\title{
TÁCTICAS EN PRÓSTATA
}

José Vicente Rodríguez

Fundación Puigvert Barcelona. Barcelona. España.

ÍN DICE/ RESUMEN

1. ¿Prostatotomía uni o bilateral?.

J. Vicente Rodríguez.

2. Tácticas en cirugía prostática ¿Q ue técnica elegir?.

J. Vicente Rodríguez.

3. RTU de próstata y tumor vesical: ¿en el mismo tiempo quirúrgico?.

J. Vicente Rodríguez.

4. Alternativas a la RTU / Incisión prostática. ¿Alguna válida?.

J. Vicente Rodríguez.

Palabras clave: Prosta totomía. Cirugía prostática. Alternativas a la RTU. Técnicas de elección.

José Vicente Rodríguez

Fundación Puigvert Barcelona.

C/ Cartagena 340-350

08025 Barcelona (España)

\section{PROSTATOTOMÍA ¿UNI O BILATERAL?}

J. Vicente Rodríguez.

La aceptación actual de la prostatotomía, se deriva de las complicaciones habidas en el pasado con la RTU en próstatas de pequeño volumen; en nuestra experiencia (1), así como en la de otros autores (2) la mayor incidencia de las esclerosis cervicales y otras complicaciones, acontecen en próstatas en las que el tejido resecado fue menor de 20 gramos.

La indicación de prostatotomía o incisión prostática está perfectamente establecida: próstatas < de 35, bilobuladas y con crecimiento cervical preferente.

La elección entre incisión bilateral (5 y 7 horas) 0 unilateral ha sido ampliamente discutida.

N osotros (3) realizamos un estudio comparativo entre 50 casos consecutivos en que realizamos prostatotomía bilateral y 50 casos de similares características en que la prostatotomía realizada fue unilateral; sus resultados quedan resumidos en la siguiente Tabla:

\begin{tabular}{|c|c|c|}
\hline RESULTADOS & $\begin{array}{c}\text { IN CISIÓ N } \\
\text { UN ILATERAL } \\
50 \mathrm{c} .\end{array}$ & $\begin{array}{c}\text { IN CISIÓ N } \\
\text { BILATERAL } \\
50 \mathrm{c} . \\
\end{array}$ \\
\hline MEJO RÍA CLÍN ICA & $84 \%$ & $92 \%$ \\
\hline $\begin{array}{l}\text { TFLUJO PRE/ POST } \\
\downarrow \text { R.P.M. (mls) }\end{array}$ & $\begin{array}{c}5,6 \mathrm{mls} / \mathrm{seg} \\
51 \mathrm{mls}\end{array}$ & $\begin{array}{c}6,5 \mathrm{mls} / \mathrm{seg} \\
58 \mathrm{mls}\end{array}$ \\
\hline M. COM PLICACION ES & $6 \%$ & $10 \%$ \\
\hline EYAC. NORMAL & $81 \%$ & $76 \%$ \\
\hline REIN TERVEN CIÓ N & $8 \%$ & $2 \%$ \\
\hline
\end{tabular}


Dado que las diferencias desobstructivas son escasas y es menor el índice de complicaciones en la incisión unilateral, consideramos al igual que otros autores (4) que esta es la técnica de rutina a elegir en los casos indicados de prostatotomía o incisión prostática; en nuestra revisión de 341 casos de prostatotomía, el $89 \%$ de las prostatotomía fueron unilaterales y solamente el $11 \%$ bilaterales. (1).

$\mathrm{N}$ uestro criterio es considerar la incisión prostática como la técnica de elección cuando está indica$\mathrm{da}$, no competitiva con la RTU y con menor morbilidad que esta, sobre todo cuando la incisión prostática se realiza con técnica unilateral.

\section{BIBUOGRAFÍA RECOMENDADA}

1. VICENTE, R.: "La prostatotomía en la HPB. ¿Por qué?”. Act. Urol. Esp.

2. TRAPASSO, J.G.; IRWIN, M.B.: "Clinical outcome and reoperation after low weight transuretral prostatectomies". J. Urol., 147: 87, 1992.

3. VICENTE, R.; SÁNCHEZ-MARTÍN, F.; PALOU, J.: "Resección versus incisión transuretral en HPB". Arch. Esp. Urol., 47: 915, 1994.

4. MOBB, G.E.; MOISEY, C.: "A long-term follow-up of unilateral blader neek incision”. Br.J. Urol. 62: 160, 1988.

\section{TÁCTICAS EN CIRUGÍA PROSTÁTICA ¿QUÉ TÉCNICA ELEGIR?}

J. Vicente Rodríguez.

\section{- Elección del paciente prostático para cirugía:}

Las indicaciones absolutas o relativas de cirugía en un momento evolutivo de la HBP, han sido ya establecidas por el International Consulting on $\mathrm{BPH}$ (1), recomendadas por las Guidelines Europeas y adaptadas por nosotros desde hace años (2).

Basándose en la información dada por el paciente: sintomatología, calidad de vida y a la observación del urólogo: tacto rectal, ecografía, flujometría y analítica, se obtienen datos que podemos agrupar en tres apartados.
1. Obstrucción: retención aguda de orina (RAO); retención crónica: residuo postmiccional (RPM) mayor de $100 \mathrm{mls}$; flujometría: Q máximo menor de 10 $\mathrm{mls} / \mathrm{seg}$.

2. Sintomatología: Valorada según cuestionario (IPSS) incluyendo calidad de vida (Q L).

3. Complicaciones: en las que incluimos infección repetida (>2-3 $x$ año), hematuria recurrente ( $>2-3 x$ año), divertículo vesical significativo, cálculos vesicales e insuficiencia renal (creatinina> 1.5).

En la Tabla I, reunimos como ejemplo nuestra experiencia en 1.037 RTUs consideradas de indicación absoluta.

Lógicamente las causas no se presentan en clínica de forma aislada, sino sumatorias: la flujometría patologica se acompaña de patología de obstrucción, la litiasis vesical se manifiesta con sintomatología severa, etc.

\section{- Elección de la técnica quirúrgica:}

Establecida la decisión quirúrgica, basándonos en las complicaciones-obstrucción-sintomatología, debemos seleccionar cual es la técnica quirúrgica idónea y la modalidad de técnica quirúrgica endoscópica si es esta la seleccionada.

Basándonos en la valoración prostática, preferentemente con el tacto rectal y la ecografía, estableceremos los tres parámetros necesarios para orientar la elección técnica:

1. Tamaño (volumen) prostático: El tacto rectal permite calcular el volumen a proximado y clasificar las próstatas en cuatro grados basada en la prominencia endorectal (2). Nosotros hemos comparado los valores medios dados por la ecografía prequirúrgica (45 mls) y el hallazgo en le acto quirúrgico (35.42 grados), 10 que se corresponde con un $85 \%$ de fiabilidad. La suma del tacto rectal y la valoración del peso calculado por ecografía nos proporciona una idea del tamaño real de la próstata. Aun siendo un parámetro importante, no es el único es más difícil realizar correctamente la RTU de una próstata pequeña que una de gran tamaño en que el volumen esté aumentado a expensas de un gran lóbulo medio. Por otro lado es mayor el índice de complicaciones en próstatas de pequeño tama- 
TABLA I.

\begin{tabular}{|c|c|c|c|c|c|}
\hline O BSTRUCCIÓ N : & $43.3 \%$ & SíN TO MAS & $42 \%$ & COM PLICA CIO N ES & $29.4 \%$ \\
\hline \multirow[t]{2}{*}{ RA O (s.u.) } & 32.2 & \multirow{3}{*}{ IPSS 20-25 } & \multirow{4}{*}{42} & IN FECCIÓN: & 3 \\
\hline & & & & HEMATURIA: & 0.8 \\
\hline \multirow[t]{2}{*}{ RPM > 100 mls: } & 23.8 & & & DIVERTÍCULO: & 6.4 \\
\hline & & \multirow[t]{2}{*}{ Q.L.5-6: } & & CALCULO V.: & 13.8 \\
\hline Q.L.5-6: & 74 & & 34.7 & IN SUF. REN AL: & 5.6 \\
\hline
\end{tabular}

ño con escaso almohadillado y corta distancia cuelloveru, que en próstatas de mayor tamaño $(2,3)$.

2. Crecimiento preferente: es un parámetro generalmente poco valorado; sin embargo lo consideramos fundamental para decidir correctamente la técnica a realizar.

El tacto rectal puede poner de manifiesto el crecimiento preferente endorectal de la próstata y su aumento en el diámetro transversal; en este caso es preferible la cirugía abierta a la RTU muy difícil en estas próstatas en forma de "montera de torero". La ecografía peroperatoria nos informa del crecimiento preferente y cuando lo hemos comparado con la visión endoscópica peropeartoria, hallamos coincidencia en el crecimiento cervical del $82.7 \%$, endouretral: $61 \%$ y endorectal: $80.6 \%$.

En próstatas con crecimiento cervical y pequeño tamaño son ideales para realizar incisión prostática y próstatas grandes pero con crecimiento preferente endouretral son tributarias de resección.
3. Forma de la próstata: está en intima relación con el crecimiento preferente y debe correlacionarse con el tamaño. La ecografía preoperatoria al compararla con el hallazgo endoscópico peroperatorio, ha representado en nuestra experiencia una fiabilidad del $69.5 \%$ en la próstata bilobulada, el 58.3 en la trilobulada y del $50.8 \%$ en próstatas con predominio del lóbulo medio. Estos datos ayudan a la elección técnica: próstata bilobulada pero pequeña orienta a realizar prostatotomía y sin embargo en una trilobulada está indicada la RTU de inicio posterior $(2,3)$.

Sumando estas características: volumen, crecimiento preferente y forma de la próstata, podemos elegir la técnica quirúrgica, según se refleja en la Tabla II.

Lógicamente este "esquema de elección técnica" puede y debe variarse según experiencia, dominio técnico, etc, pero pretende reflejar el consenso de mínimos útil para la mayoría de urólogos.

TABLA II.

\begin{tabular}{|l|l|l|l|}
\hline TÉCN ICA & TAMAÑO & CRECIMIEN TO & FORM A \\
\hline PRO STATO TO M ÍA & $<30 \mathrm{gr}$ & Cervical & Bilobulada \\
\hline RTU IN ICIO ANT. & $30-60 \mathrm{gr}$ & $\begin{array}{l}\text { Endouretral } \\
\text { Endovesical } \\
\text { Central } \\
\text { Lóbulo medio }\end{array}$ & Bilobulada \\
RTU IN ICIO POST. & $30-60 \mathrm{gr}$ & $\begin{array}{l}\text { Endorectal } \\
\text { Lateral }\end{array}$ & $\begin{array}{l}\text { Bilobuladada } \\
\text { Trilobulada }\end{array}$ \\
\hline CIRUG ÍA ABIERTA & $>60 \mathrm{gr}$ & & \\
\hline
\end{tabular}




\section{BIBUOGRAFÍA RECOMENDADA}

1. CHATELAIN, C.H.; DENIS, L.; FOO, J.K.T. y cols.: "Recommendations of the International Scientific Committee: evaluation and treatment of LUTS in older men". Benign prostatic hyperplasia. Editors C. Chatelain, L. Denis, y cols. Health Publication, 519, 2001.

2. VICENTE, J.: "Estrategias terapéuticas quirúrgicas en Fundación Puigvert en HBP 2001”. Ed. J. Vicente. Ed. Acción Médica, 233, 2001.

3. VICENTE, J.: "La cirugía transuretral de la próstata". Hipertrofia Prostática Benigna. Ed. O. Leiva, J. Angulo, J. González. Editorial Luzan, 475, 2003.

\section{RTU DE PRÓSTATA Y TUMOR VESICAL: ¿EN EL MISMO TIEMPO QUIRÚRGICO?}

J. Vicente Rodríguez.

La coincidencia clínica de tumores vesicales y próstata benigna obstructiva, plantean un problema estratégico en relación a su tratamiento endourológico: coincidente o diferido.

Trabajos clásicos experimentales (1), reactivados recientemente (Van der Mesden), parecen demostrar la implantación celular en áreas denudadas por la cirugía endoscópica.

Ello justificó desaconsejar la resección simultánea del tumor vesical y próstata, dado el riesgo de implantación de células tumorales en la superficie denudada de la celda prostática.
A utores de prestigio (2) recomiendan realizar la resección tumoral y tres semanas después la de próstata (previa revisión endoscópica vesical)

Trabajos comparativos (3) y nuestra propia experiencia, que se muestra en la Tabla I, no muestran diferencias significativas en el número de recidivas, tiempo de aparición ni su grado / estadio tumoral, en casos comparativos en que se ha realizado resección de tumor vesical aislado versus RTU de tumor vesical y próstata en el mismo acto quirúrgico.

Estos resultados nos ha permitido realizar y nos permite aconsejar la RTU simultánea del tumor vesical y de la próstata en los casos de coincidencia clínica de ambos.

\section{BIBUOGRAFÍA RECOMENDADA}

1. SOLOWAY, M.S.; MASTERES, S.: "Urothelial suceptability to tumor cell implantation. Influence of cauterization”. Cancer, 46: 1158, 1980.

2. BAGLEY, D.H.; LYON, E.S.; HUFFMAN, J.L.: "Transurethral resection of bladder tumors". Urologic Endoscopy a manual and atlas. Ed. DH. Bagley, JL Huffman, ES Lyon. Edit: Little, Brown and company Boston, 131, 1985.

3. LAOR, E.; GRABSTALD, H.; WHITMORE, W.F.: "The influence of simultaneous resction of bladder tumors and prostate on the recurrence of prostatic uretral tumors". J. Urol., 126: 171, 1981.

4. VICENTE, J.; CHECHILE, G.; PONS, R. y cols.: "Tumor recurrence in prostatic urethal following simultaneous resction of bladder tumor and proatate". Eur. Urol., 15: 40, 1988.

TABLA I.

\begin{tabular}{|l|c|c|}
\hline RECURREN CIAS EN CELDA PR & 100c. RTU TM VESICAL & 100c. RTU TM V + RTU PRO ST \\
\hline NÚMERO RECURREN CIAS & 12 & 14 \\
\hline TIEMPO A PARICIÓN & 28 meses & 36 meses \\
\hline GRADO / ESTADIO & G2:9c T1:9c. & G2: 8c T1:9c. \\
\hline
\end{tabular}




\section{ALTERNATIVAS A LA RTU / INCISIÓN PROSTÁTICA: ¿ALGUNA VÁUDA?}

J. Vicente Rodríguez.

Para el urólogo practico la táctica entre las alternativas es poder discriminar:

1. Q ue alternativas debe desechar en su actividad clínica.

2. Que alternativas puede aceptar como válidas, dependiendo su inclusión en la práctica según sus prioridades o preferencias.

3. Que alternativas no puede ni descartar ni aceptar por falta de estudios o tiempo de seguimiento suficiente.

La mayoría de las alternativas no han soportado el paso del tiempo (valoración a 1 a 5 años), ni la exigencia científica (metaanálisis, estudios randomizados $(1,2)$ ); nosotros hemos realizado un riguroso estudio comparativo entre alternativas y cirugía endoscópica, basándonos en 30 estudios randomizados y 28 clínicos relevantes incluyendo la experiencia personal (3).

Basándonos en los datos obtenidos, se puede establecer:

1- ALTERNATIVAS DESCARTABLES: láser/ coagulación (VLAP), TUNA, láser intersticial, láser de contacto y electrovaporización.
La valoración entre la RTU/ Incisión y estas alternativas viene resumida y gráficamente simplificada en la Tabla I.

Para descartar estas alternativas debemos valorar comparativamente:

- Mejoría subjetiva: disminución de la sintomatología similar en las alternativas y la cirugía prostática.

- Mejoría objetiva: exceptuando la electrovaporización, la elevación postoperatoria del Q max. es el doble en la cirugía prostática que en las alternativas.

- Necesidad de retratamiento: representa el doble y triple en las alteraciones que en la cirugía endoscópica (excepto en la E.V); realmente la RTU/ Incisión resulta ser la solución al fracaso de las alternativas.

- Síntomas irritativos post tratamiento: son superiores en todas las alternativas excepto en TUNA y Láser Intersticial que son similares a la RTU; el disconfort es altamente significativo post V LAP y Electrovaporización.

- Días de sonda uretral : los días de sonda son el triple que en la RTU, en el VLAP y Láser Intersticial aunque es menor en el TUN A y similar en el láser de contacto.

TABLA I.

\begin{tabular}{|l|l|l|l|l|l|}
\hline RTU/ TUIP & VLAP & TUNA & L. INT & L. CONT & E. VAPOR \\
SÍNTOMAS & $=$ & $=$ & $=$ & $=\downarrow$ & $=$ \\
FLUJO & $\downarrow$ & $\downarrow \downarrow$ & $\downarrow \downarrow$ & $\downarrow$ & $=$ \\
Re-TRAT & $\uparrow \uparrow$ & $\uparrow \uparrow \uparrow$ & $\uparrow \uparrow$ & $\uparrow \uparrow$ & $\uparrow$ \\
S. IRRITATIVOS & $\uparrow \uparrow$ & $=$ & $=$ & $\uparrow$ & $\uparrow \uparrow$ \\
DIAS S/ N & $\uparrow \uparrow \uparrow$ & $\downarrow$ & $\uparrow \uparrow$ & $=\downarrow$ & $=$ \\
AN ESTESIA & $=$ & $\downarrow$ & $=\downarrow$ & $=$ & $=$ \\
\hline
\end{tabular}


- Necesidad de anestesia: la anestesia general 0 intradural es necesaria en todas estas a lternativas excepto en TUNA y como inducción en el Láser Intersticial.

2.- A LTERN ATIVA S A CEPTA BLES: condicionadas a circunstancias o preferencias: TUMT, Ho Láser. PVP. Prótesis.

Estas alternativas son válidas en situaciones clínicas especiales: alteraciones de la coagulación, riesgo quirúrgico elevado, preferencias del paciente: negativa a cirugía convencional, disminución de riesgo en eyaculación retrógrada o del urólogo: rechazo por coste elevado, incentivos sa nitarios etc.

TUMT.- Válida como alternativa en casos en que la cirugía está condicionada (riesgo quirúrgico elevado, alteración hematológica etc), dado que su técnica es sencilla, se realiza con anestesia local y sin hospitalización. Sin embargo no es válida en "todos los casos", dado que obtiene menor respuesta objetiva, mayor índice de retratamiento y días de sonda uretral post, que la RTU. En las Guidelines Europeas: recomendado en pacientes sin respuesta a la medicación y descartan la cirugía.

Holmium Láser- Sus resultados son comparables y con menor morbilidad que la RTU (4). Sin embargo su curva de aprendizaje es superior al de la cirugía endoscópica, el tiempo quirúrgico más prolongado y su coste muy elevado; por ello la decisión de su aceptación debe ser tomada de forma individual por el urólogo 0 los centros sanitarios implicados. En las EAU G uidelines recomendada como verdadera alternativa

P.V.P. La fotovaporización con láser KTP del alto voltaje (80W) consigue respuesta subjetiva/ objetiva con similar tiempo quirúrgico y morbilidad (irritativos: 20\%, RAO postop:5\%) al de la cirugía endoscópica (5) y se mantienen a un seguimiento de 5 años (Malek, J Urol 2003.169. A bst.1457).

Sin embargo existen pocos trabajos en la bibliografía y la experiencia con el "láser verde" en nuestro medio es escasa. No valorada en las EAU Guidelines.

Prótesis Endouretrales- Las temporales como alternativa a la sonda uretral a permanencia y las per- manentes a la cirugía prostática, cuando esta no es posible (alto riesgo anestésico quirúrgico). Su capacidad desobtructiva es similar a la de la RTU, pero es alta la tasa de complicaciones, relevante el índice de retratamiento y porcentaje no despreciable de necesidad (con dificultad), de extraer la prótesis. (6). Por estas razones la colocación de la prótesis queda limitada a casos muy seleccionados. Solo recomendadas en las EAU Guidelines en pacientes de alto riesgo quirúrgico.

\section{3.- A LTERN ATIVAS CO N DICIO N ADAS.}

La falta de tiempo de seguimiento y el número reducido de estudios con rigor científico o cohorte de pacientes suficientes, condicionan la aceptación o rechazo de supuestas alternativas como Rotoreset, W IT et (3).

La inyección transuretral/ intraprostática de etanol ha sido recientemente evaluada por un grupo multricéntrico europeo (7) con seguimiento a un año: mejoría subjetiva: $50 \%$ pero aumento del flujo de solo $35 \%$, sintomatología irritativa post. del $26 \%$ y reoperación (RTU) al año del 7\%. Se necesita mayor seguimiento para decidir su inclusión.

La RTU con bisturí bipolar, no puede considerarse como alternativa sino como modificación o mejora de la RTU convencional (8): corte más "limpio", no reabsorción, menor tiempo quirúrgico (9).

N osotros consideramos más interesantes los avances técnicos orientados a facilitar la RTU reduciendo su morbilidad (asas ovaladas o bipolares, fuentes de energía no eléctrica o por pulsos, regulación digitalizada de presión intracavitaria, etc), que invertir en el desarrollo de Alternativas siempre comparadas con la resección transuretral, siendo además esta la solución en casos de su fracaso.

\section{BIBUOGRAFÍA RECOMENDADA}

1. MADERSBACHER, S.; MARBERGER, M.: "Is transurethral resection of the prostate still justified?". BJU Int., 83: 227, 1999.

2. TUBARO, A.; VICENTINI, C.; RENZETTI, R. y cols.: "Invasive and minimally treatment modalities for lower urinary tracts symptoms: What are the relevant differences in randomized controlled trials?". Eur. Urol., 38: 7, 2000. 
3. VICENTE, J.R.: "Visión actual y crítica de las alternativas a la cirugía transuretral de la hiperplasia benigna de próstata". Arch. Esp. Urol., 56: 102, 2003.

4. TAN, A.H.; GILLING, P.S.: "Holmium Laser prostatectomy current techniques". Urology, 60: 152, 2002.

5. TE, A.E.; TERRENCE, R.; MALLOY, y cols.: "Photoselective vaporizatión of the prostate for the treatment of bening prostatic hiperplasia: 12-month results from the first united states multicenter prospective trial". J. Urol., 172: 1404, 2004.

6. VICENTE, J.R.: "La cirugía transuretral de la próstata. En hipertrofia Prostática benigna". Id. O. Leiva, J. Angulo. J. Gonzalez, 24: 475, 2003.

7. GRISE, P.; PLANTE, M.; PALMER, J. y cols.: "Evaluation of the transurethral Ethanol ablation of the prostate (TEAP) for syntomatic BPH: A European multi-center evaluation". Eur. Urol., 46: 496, 2004.

8. BARRERO, R.; SÁNCHEZ, J.M.; BLASCO, B. y cols.: "Resección transuretral de próstata con bisturí bipolar". Arch. Urol. Esp., 28: 147, 2004.

9. ENDT-NORDAHAL, G.; HÄCKER, A.; REICH, O. y cols.: "The Vista System: a new bipolar resection disector endourological procedures: comparation with conventional resectoscope". Eur. Urol., 46: 586, 2004. 